\title{
Synonym comprehension and the generality of categorization models
}

\author{
DOUGLAS J. HERRMANN, THEODORE J. PAPPERMAN, \\ and ALAN D. ARMSTRONG \\ Hamilton College, Clinton, New York 13323
}

\begin{abstract}
When subjects classify a two-word display as representing the "same" category or two "different" categories, semantic similarity between the words facilitates "same" decisions but impedes "different" decisions. The present research investigated whether the similarity effect observed for categorization would also be found in synonym comprehension; that is, the task of deciding whether two words are or are not synonymous. Experiment 1 found that an increase in semantic similarity between two "partial" synonyms facilitated synonym response latency. Experiment 2 found that an increase in the similarity between two nonsynonyms impeded nonsynonym response latency. Thus, the similarity effect on categorization latency and on synonym comprehension resemble each other. Moreover, models of the categorization process that account for the similarity effect on categorization appear to be applicable to synonym comprehension.
\end{abstract}

Several theories have been developed in recent years to account for the comprehension of categorical relationships (Smith, 1977). Thus, it seems propitious to inquire about the semantic generality of category models. Will the pattern of results be similar to that observed for category comprehension if the comprehension task is based on some other semantic relationship, such as synonymy? If the pattern of results were found to be similar across comprehension of category membership and synonymy, then semantic memory models that account for categorization would acquire greater generality because they could also be applied to synonymy.

Theoretical similarities between categorical relationships (superordinate-subordinate; two coordinates) and synonymy suggest that synonym comprehension might resemble category comprehension. Both categorical relationships and synonymy involve approximate agreement in denotative meaning, except that only a subset of such meaning is relevant to categorical relationships (Herrmann, in press; Rosch \& Mervis, 1975). Given these similarities, the present research investigated whether or not two robust findings concerning category comprehension extend to synonym comprehension; that is, the decision that two words do or do not possess the same meaning.

Among the many studies involving category terms, two kinds of investigations have been particularly

Part of this research was funded by a National Science Foundation grant (to the first author) for an undergraduate research training program (in which the second author participated). The authors thank Harry Hersh and Robert J. Sternberg for their very helpful comments on an earlier draft of the article. Requests for reprints should be sent to Douglas J. Herrmann, Department of Psychology, Hamilton College, Clinton, New York 13323. prominent. First, categorical research has often attempted to scale the similarity relationships between members of a category or different categories. For example, the scaling technique of hierarchical clustering (Johnson, 1967), when applied to measures of similarity between terms, has been found to yield an interpretable arrangement of terms at varying distances in a hierarchy when these terms represented the same category (Fillenbaum \& Rapoport, 1971) or different categories (Collen, Wickens, \& Daniele, 1975; Herrmann, Shoben, Klun, \& Smith, 1975). Scaling procedures that are not hierarchical, but rather multidimensional, have also been successful in analyzing and depicting similarity relationships of category terms in a plausible spatial representation (Caramazza, Hersh, \& Torgerson, 1976; Henley, 1969; Rips, Shoben, \& Smith, 1973).

Categorical research has also attempted to relate the "semantic distance" extracted from similarity measures by scaling analyses to the latency to make categorical judgments. For example, when subjects classified two words as belonging to the same category (Caramazza et al., 1976; Rips et al., 1973), response latency was found to be faster when the semantic distance separating members of the pair was small (hawk-agle) than when members of the pair were distant (hawk-bluejay). Conversely, when subjects classified two words as belonging to different categories (Herrmann et al., 1975), response latency was found to be slower when the semantic distance within a pair was close (tulip-oak) than distant (tulip-chair). In summary, the effect of semantic distance on samecategory decisions is opposite to its effect on differentcategory decisions.

The present study investigated the effect of scaled semantic distance on synonym comprehension in two experiments in a manner analogous to category 
investigations. These experiments collected similarity data for synonyms (Experiment 1) and nonsynonyms (Experiment 2), applied scaling techniques (described below) to the similarity data, and varied the semantic distance obtained from the scaling solution in a synonym comprehension task. In Experiment 1, two "synonym sets" (cf. Weinreich, 1962) of 11 words each were rated for similarity in meaning and subjected to a hierarchical clustering analysis. Subsequently, synonym comprehension latency was examined for synonym pairs composed of two words that were close or distant in the hierarchical analysis. (It should be noted that distance was dichotomized here only for simplicity and that the full range of distance values could just as well have been used in a correlational analysis of latency and distance.) The method and results of the scaling analysis are presented first, followed by the method and results of the synonym comprehension experiment.

\section{EXPERIMENT 1}

\section{Method: Hierarchical Clustering Analysis}

Subjects. A group of 25 people, consisting of students, businessmen, housewives, and professors, filled out a synonymevaluation questionnaire without pay.

Materials and Procedure. A booklet was constructed of an instruction page and two sections of 11 pages each. One section presented stimuli from a synonym set referred to here as appetitive emotions [i.e., covet, desire, dream, hope, long (for), lust, need, strive, want, wish, and yearn] and one for a synonym set of mental ability terms (i.e., bright, clever, cunning, intelligent, quick, sharp, sly, smart, tricky, witty, and wise). Each stimulus page was headed by one of 11 different stimulus words, below which was a column of the remaining 10 words (in random order) with a line alongside of each word. Half of the booklets presented the appetitive emotions first, and half presented the mental ability terms first. The directions asked the subject to judge the similarity between each of the 10 stimulus words on each page and the stimulus word at the top of the page on a 7-point scale, with 7 representing identical meanings and 1 representing very dissimilar meanings. The subject recorded his/her judgments on the line alongside each comparison word. The questionnaire was completed at home by the subject. The means of similarity ratings were analyzed by the hierarchical clustering program (Johnson, 1967) of OSIRIS.

\section{Results and Discussion}

The results of the hierarchical clustering analyses for the appetitive emotions are shown in the upper half of Figure 1 and for mental ability terms in the lower half of the figure. Inspection of either solution reveals that the hierarchical clustering analyses arranged the terms in an intuitively satisfactory manner. With the clustering analysis of the two synonym sets completed, an experiment was designed to investigate the effects of semantic distance on synonym comprehension. Subjects were presented with synonym pairs in which the two words were closely or widely separated in the hierarchical solutions shown in Figure 1.

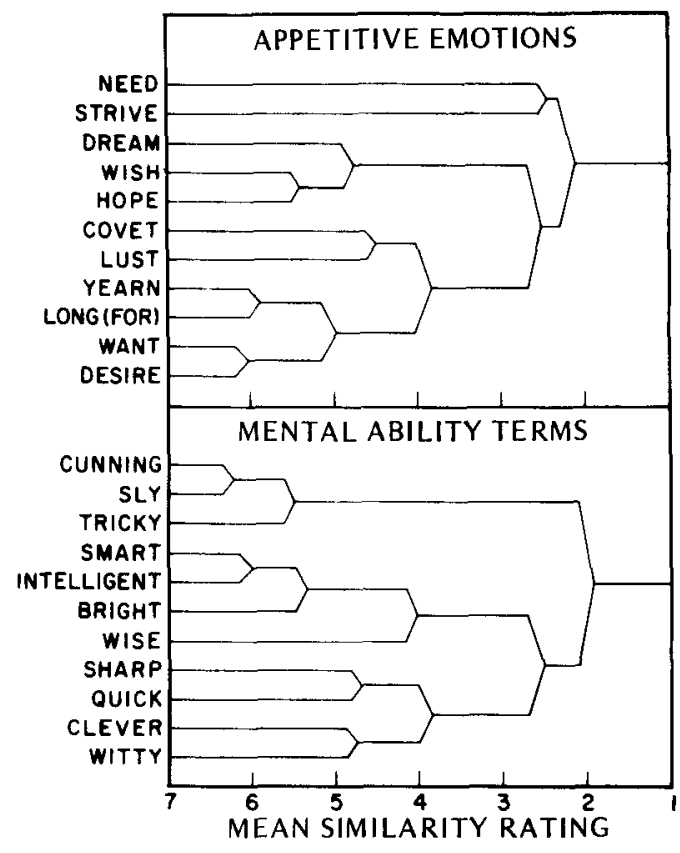

Figure 1. A hierarchical clustering solution for two synonym sets as a function of mean similarity rating (where ratings of one and seven represent low and high similarity, respectively).

\section{Method: Synonym Comprehension Task}

Subjects. Fifteen undergraduates from Hamilton and Kirkland colleges served in the experiment and were paid $\$ 2$ each.

Stimulus materials. Sixteen close pairs and 16 far pairs were constructed with 8 pairs in either distance condition coming from the two synonym sets in Figure 1. Close pairs contained two words that originated from one of the intermediate clusters in the figure [i.e., need-strive, dream-wish-hope, and covet-lust-yearn-long (for)-want-desire; cunning-sly-tricky, smart-intelligent-bright-wise, and sharp-quick-clever-witty]. Far pairs contained two words drawn from different intermediate clusters in the same synonym set. To insure that each word was used approximately equally often over all 16 pairs from a set, only 8 words of the 11 words in a set were used for constructing stimuli. However, a particular combination of words in a pair was used only once. Since pilot research had indicated that repeated presentation of just two synonym sets leads to "semantic satiation" and incomplete processing of the synonym stimuli (Lambert \& Jakabovitz, 1960), 32 synonym pairs from two other synonym sets (terms of "possession" and "loyalty") were included as filler material in the presentation series of appetitive emotions and mental ability terms. Thirty-two nonsynonym pairs consisted of an appetitive emotion paired with a mental ability term or a "possession" term paired with a "loyalty" term.

Apparatus. A three-field tachistoscope (Iconix, Model 6137) was used to present stimulus pairs. The words in a pair were typed in uppercase, centered one above the other. Responses were made with hand-held microswitches, which stopped a response timer (Iconix response buffer, Model 6237) accurate to $\pm 1 \mathrm{msec}$.

Procedure. The subject was directed to classify a two-word display as containing synonyms (by pressing a microswitch held in the hand designated for synonym responses) or nonsynonyms (by pressing the microswitch in the other hand, designated for nonsynonym responses). Hand dominance and response type was counterbalanced across subjects. Synonymy 
was defined for the subject as sameness in meaning and explained to be related to substitutability in writing (cf. Rubenstein \& Goodenough, 1965). Directions stressed accuracy over speed in responding. Subjects were tested in two sessions, each consisting of six practice trials and 64 test trials, randomly ordered.

For the statistical analysis, the data for the two sessions were combined. Neither the nonsynonym latencies nor the latencies for filler material were analyzed, since these conditions were irrelevant to the goals of the experiment.

\section{Results}

Mean latency of correct synonym responses to pairs from the appetitive emotions and from the mental ability terms was $1,183 \mathrm{msec}$ for close pairs and $1,476 \mathrm{msec}$ for far pairs. An analysis of variance including items as a random factor (Clark, 1973) confirmed that close pairs were classified more rapidly than far pairs $\left[\min \mathrm{F}^{\prime}(1,43)=9.87, \mathrm{p}<.01\right]$. The mean percent of synonym pairs given nonsynonym responses was $7.9 \%$ for close pairs and $21.3 \%$ for far pairs $\left[\min F^{\prime}(1,19)=4.18, .10>p>.05\right]$. The mean percentage of synonym responses made to the nonsynonym pairs was $9.9 \%$.

\section{Discussion}

The results showed that hierarchical distance affected latency of synonym comprehension for synonyms of appetitive emotions and of mental ability terms. This experiment, therefore, reproduced with synonyms the essential aspects of the Rips et al. (1973) findings on semantic distance and latency of same-category decisions (replicated and extended by Caramazza et al., 1976). Semantic distance from a scaling solution appears to affect latency in the same manner for categories and synonym sets.

The present results also reproduced previous findings from research on synonym comprehension. Esposito and Pelton (1969) and Fillenbaum (1964) found synonym response latency was faster for two synonymous words that were "close" in meaning than for synonyms "far apart" in meaning. Experiment 1, therefore, extends the findings of these investigators for similarity in meaning in general to the more narrowly defined semantic distance extracted by scaling techniques.

\section{EXPERIMENT 2}

A second finding from categorization research, described earlier, is that semantic distance impedes the decision that two words belong to different categories (Herrmann et al., 1975; cf. Schaeffer \& Wallace, 1970). If synonym comprehension involves processes similar to those in category comprehension, then the latency to determine that two words are not synonymous should depend on the semantic distance between two nonsynonyms. In particular, nonsynonym response latency should be longer for similar nonsynonyms than dissimilar nonsynonyms.
Experiment 2 tested this prediction in a manner similar to that done for categorization by Herrmann et al. (1975). The similarity between a group of nonsynonyms was first obtained by having subjects inspect the 80 synonym sets in the Haagen (1949) norms and then arrange them into groups where the sets in a group shared some aspect of meaning. The frequency by which any two sets grouped together was then treated like any other similarity measure (e.g., ratings) and subjected to a hierarchical clustering analysis. The grouping procedure was used in lieu of the rating procedure of Experiment 1 because ratings for all possible pairs of the 80 synonym sets ( 3,160 pairs) would have required several hours of a subject's time, whereas the grouping procedure for the 80 sets required less than $1 \mathrm{~h}$.

The effect of semantic distance in the clustering solution on nonsynonym response latency was subsequently examined in a synonym comprehension task. Nonsynonym pairs contained two words from different synonym sets that were close or distant in the hierarchical clustering analysis. As in Experiment 1, the method and results of the hierarchical clustering analysis are presented first, followed by the method and results of the synonym comprehension task.

\section{Method: Hierarchical Clustering Analysis}

Subjects. Forty-five Hamilton and Kirkland college undergraduates served in the experiment and were paid $\$ 1.50$ each.

Materials. Eighty synonym sets were drawn from the Haagen (1949) synonym norms. These norms present 80 synonym sets composed of one "standard" word and five "comparison" words that are approximately synonymous with the standard word. The present experiment used each group of comparison words as synonym sets for the clustering task and the standard word as labels to refer to synonym sets in the hierarchical clustering analysis. Each synonym set of five words was typed on $3 \times 5$ in. index cards.

Procedure. Each subject was given the deck of 80 stimulus cards and directed to sort them into two or more piles in which the synonym sets in a pile were similar in meaning.

Analysis. The particular groupings by each subject were recorded and the data for all 45 subjects was analyzed by the OSIRIS hierarchical clustering computer program (based on Johnson, 1967). Since neither OSIRIS nor any other clustering routine available to us would permit more than 75 cases, four separate analyses were performed; each analysis was done on a different combination of 75 synonym sets out of the total 80 synonym sets such that the 5 sets excluded from each analysis were different across the four analyses. Because the overlapping parts of the different solutions (diameter method) yielded almost identical hierarchical structures, the four solutions were integrated by eye into a composite hierarchical representation for all 80 synonym sets.

\section{Results}

The composite representation of the hierarchical clustering analyses for the 80 synonym sets is presented in Figure 2, Panels a and b. The division into two panels was done in a manner that did not break up part of the hierarchical structure, in that Panels a and b represent terminal groupings. Each word shown in the figure serves as a label for a synonym set of five words. The 


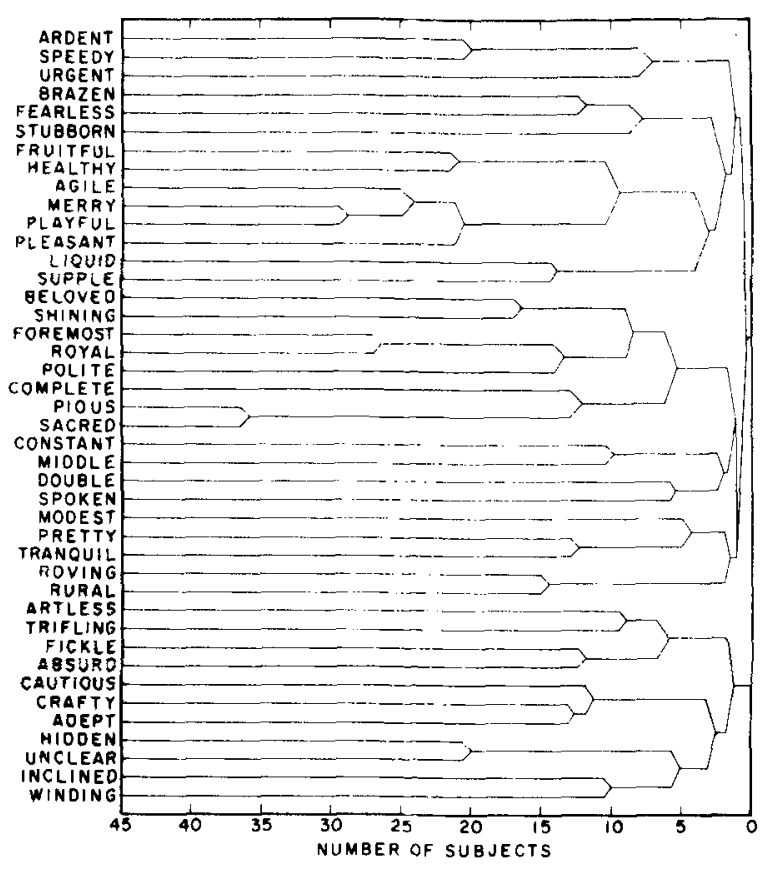

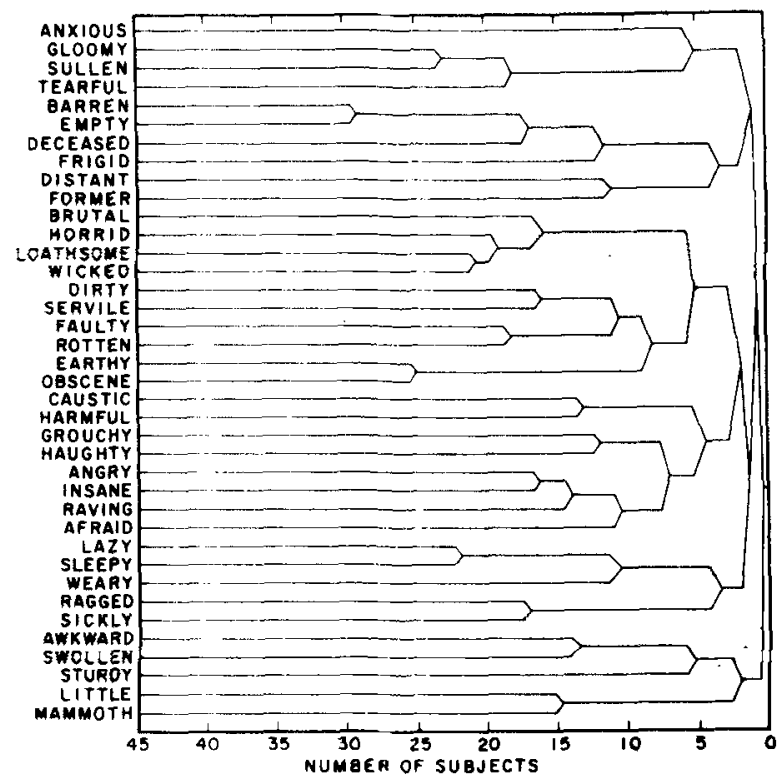

Figure 2. Panels a (left) and $b$ (right) of a composite tree graph based on hierarchical clustering analy sis applied to the groupings of 80 synonym sets from the Haagen (1949) norms by 45 subjects.

words comprising a set are listed alongside each of the present labeis in the Haagen (1949) norms. Inspection of the hierarchical clustering solution in Figure 2 reveals that the hierarchy contained three large or "major" clusters and that many of the smaller clusters in each major cluster intuitively would be expected to be found in this clustering analysis.

The solution depicted in Figure 2 demonstrates that semantic distance varies between synonym sets in a manner like that found for different categories (cf. Collen et al., 1975; Herrmann et al., 1975). Given this evidence that synonym sets can be scaled at varying semantic distances, it became appropriate to ask whether such distance affects classification of nonsynonyms in a synonym comprehension task.

\section{Method: Synonym Comprehension Task}

Subjects. Twelve students from Hamilton and Kirkland colleges performed the synonym comprehension task and were each paid $\$ 2$. None of these subjects served in the clustering investigation.

Materials. Thirty nonsynonym pairs contained two words that were either "close" (is pairs) or "far" (15 pairs) in the hierarchy of Figure 2. A close nonsynonym pair consisted of one word from each of two different synonym sets that were connected by a single node in Figure 2 . A far nonsynonym pair consisted of one word from each of two synonym sets that did not belong to the same major cluster, defined as one of the three largest clusters in Figure 2.

Synonym pairs were constructed from other words in the Haagen (1949) norms not used in nonsynonym pairs. Across synonym and nonsynonym pairs, no words occurred twice but some synonym sets were presented twice (equally often for close and far nonsynonym pairs). Also, mean printed frequency from the Kučra and Francis (1967) norms was equated across conditions: 13.6 for synonym pairs, 13.5 for both close and far nonsynonym pairs.

Procedure. The procedure was identical to that in the preceding experiment except that a subject was tested in only a single session, which consisted of 10 practice pairs and 60 test pairs. In the statistical analysis, only the nonsynonym latencies were analyzed since the synonym responses were irrelevant to the goals of the experiment.

\section{Results}

Mean latency of correct nonsynonym responses was $1,757 \mathrm{msec}$ for close pairs and $1,508 \mathrm{msec}$ for far pairs. An analysis of variance including items as a random factor (Clark, 1973) confirmed that far pairs were classified more rapidly than close pairs $\left[\min F^{\prime}(1,30)=7.41, \mathrm{p}<.05\right]$. More close nonsynonyms were classified as synonyms $(16.1 \%)$ than far pairs $(2.8 \%) \quad\left[\min F^{\prime}(1,35)=5.58, \mathrm{p}<.05\right]$. The mean percentage of nonsynonym responses made to synonym pairs was $3.6 \%$.

\section{Discussion}

The results showed that an increase in hierarchical distance between words in nonsynonym pairs decreased nonsynonym response latency. This result is essentially the same as that obtained in the categorization task by Herrmann et al. (1975), who found that differentcategory decision latency decreased with the distance between categories in a hierarchical analysis of the categories in the Battig and Montague (1969) norms (cf. Schaeffer \& Wallace, 1970). The results for Experiment 2 are also consistent with Sabol and De Rosa's (1976) finding that nonsynonym response 
latency was greater for two words related by opposition, due to being antonyms, than for two unrelated words.

It should be noted that some pairs of synonym sets in the clustering solution of Experiment 2, described as nonsynonyms, nevertheless seem as synonymous as some of the synonym pairs in Experiment 1 (e.g., merry-playful in Figure 2 vs. witty-sly in Figure 1). Since such pairs are obviously in the region of semantic similarity where synonymy and heteronymy converge (cf. Herrmann, in press), it may seem surprising that subjects generally classified these pairs in the expected category of synonyms and nonsynonyms. The high percentage of these classifications, though, becomes reasonable when context is taken into account (Tversky, 1977). Nonsynonyms in Experiment 1 were clearly not synonymous, leading subjects to judge marginal synonyms as synonymous. Similarly, synonyms in Experiment 2 were highly similar, making nonsynonym classifications of the less similar, close nonsynonyms very likely. Further research is needed to systematically determine how responses to borderline synonyms vary with different semantic contexts.

In conclusion, the results of Experiments 1 and 2, along with the findings of similar investigations (Esposito \& Pelton, 1969; Fillenbaum, 1964; Sabol \& De Rosa, 1976), demonstrate that semantic similarity facilitates same-meaning decisions (i.e., synonym responses) and impedes different-meaning decisions (i.e., nonsynonym responses). Since the inverse relationship between semantic similarity and response type has also been obtained in many category investigations (Smith, 1977), it appears that categorization models that account for this inverse relationship also apply to synonym comprehension (see models of Collins \& Loftus, 1975; Gellatly \& Gregg, 1977; Glass \& Holyoak, 1974/1975; Meyer \& Schvaneveldt, 1976; Smith, Shoben, \& Rips, 1974; McCloskey \& Glucksberg, Note 1). Since all these models can account for the inverse influence of similarity on the two response types, however, more research will be necessary to determine which categorization model is most applicable to synonym comprehension. Nevertheless, categorization models apparently possess greater semantic generality than was originally proposed by the developers of these models.

\section{REFERENCE NOTE}

1. McCloskey, M., \& Glucksberg, S. Test of a feature comparison model. Paper presented at the American Psychological Association, Washington, D.C: September 1976.

\section{REFERENCES}

Battig, W. F., \& Montague, W. E. Category norms for verbal items in 56 categories: $A$ replication and extension of the Connecticut category norms. Journal of Experimental Psychology Monographs, 1969, 80, 1-46.

Caramazza, A., Hersh, H., \& Torgerson, W. S. Subjective structures and operations in semantic memory. Journal of Verbal Learning and Verbal Behavior, 1976, 15, 103-107.

Clark, H. H. The language-as-a-fixed-effect fallacy: A critique of language statistics in psychological research. Journal of Verbal Learning and Verbal Behavior, 1973, 12. 335-359.

Collen, A., Wickens, D. D., \& Daniele, L. The interrelationships of taxonomic categories. Journal of Experimental Psychology: Human Learning and Memory, 1975, 1 , 629-633.

Collins, A. M., \& Loftus, E. F. A spreading-activation theory of semantic processing. Psychological Review, 1975, 82, 407-428.

Esposito, N. J., \& Pelton, L. H. A test of two measures of semantic satiation. Journal of Verbal Learning and Verbal Behavior, 1969, 8, 637-644.

Fillenbaum, S. Semantic satiation and decision latency. Journal of Experimental Psychology, 1964, 68, 240-244.

Fillenbaum, S., \& Rapoport, A. Structures in the subjective lexicon. New York: Academic Press, 1971.

Gellatly, A. R. H., \& GReGG, V. H. Intercategory distance and categorization times: Effects of negative-probe relatedness. Journal of Verbal Learning and Verbal Behavior, $1977,16,505-518$.

Glass, A. L., \& Holyoak, K. J. Alternative conceptions of semantic memory. Cognition, 1974/75, 3, 313-339.

HAAGEN, H. S. Synonymity, vividness, familiarity, and association, value ratings of 400 pairs of common adjectives. The Joumal of Psychology, 1949, 27, 244-248.

HENLEY, N. A. A psychological study of the semantics of animal terms. Journal of Verbal Leaming and Verbal Behavior, 1969, 8, 176-184.

HeRrmanN, D. J. An old problem for the new psychosemantics: Synonymy. Psychological Bulletin, in press.

Herrmann, D. J., Shoben, E. J., Klun, J. R., \& Smith E. E. Cross-category structure in semantic memory. Memory \& Cognition, 1975, 3, 591-594.

Johnson, S. C. Hierarchical clustering schemes. Psychometrika, 1967. 32, 241-254.

KuĊera, H., \& Francis, W. N. Computational analysis of present day American English. Providence: Brown University Press, 1967.

LAMBERT, W. E., \& JAKobovits, L. A. Verbal satiation and changes in the intensity of meaning. Journal of Experimental Psychology, 1960, 60, 376-383.

Me yer, D. E., \& Schvaneveldt, R. W. Meaning, memory structure and mental processes. Science, 1976, 192, 27-33.

Rips, L. J.. Shoben, E. J., \& Smith, E. E. Semantic distance and the verification of semantic relations. Journal of Verbal Learning and Verbal Behavior, 1973, 12, 1-20.

Rosch, E., \& Mervis, C. B. Family resemblances: Studies in the internal structure of categories. Cognitive Psychology, 1975, 7, 573-605.

Rubenstein, H., \& Goodenough, J. B. Contextual correlates of synonymy. Communications of the Association for Computing Machinery, 1965, 8, 627-633.

SABol, M. A., \& De Rosa, D. V. Semantic encoding of of isolated words. Journal of Experimental Psychology: Human Learning and Memory, 1976, 2, 58-68.

Schaefer, B., \& Wallace, R. The comparison of word meanings. Journal of Experimental Psychology, 1970, 86, 144-152.

SMITH, E. E. Theories of semantic memory. In W. K. Estes (Ed.), Handbook of learning and cognitive processes (Vol. 5). Hillsdale, N.J: Lawrence Erlbaum, 1977. 
Smith, E. E., Shoben, E. J., \& Rips, L. J. Structure and process in semantic memory: A featural model for semantic decisions. Psychological Review, 1974, 81, 214-241.

TVErsky, A. Features of similarity. Psychological Review. 1977, 84, 327-353.

WeINREICH, V. Lexicographic definition in descriptive semantics. In F. W. Householder \& S. Saporta (Eds.).
"Problems in Lexicography." International Joumal of American Linguistics, 1962, 28, 25-43.

(Received for publication September 12, 1977; accepted November $30,1977$. 